Publ. Mat. 60 (2016), 485-500

DOI: 10.5565/PUBLMAT_60216_06

\title{
INTEGRAL RESTRICTION FOR BILINEAR OPERATORS
}

\author{
Weiren Zhao, Meng Wang, and Guoping Zhao
}

\begin{abstract}
We consider the integral domain restriction operator $T_{\Omega}$ for certain bilinear operator $T$. We obtain that if $\left(s, p_{1}, p_{2}\right)$ satisfies $\frac{1}{p_{1}}+\frac{1}{p_{2}} \geq \frac{2}{\min \{1, s\}}$ and $\|T\|_{L^{p_{1}} \times L^{p_{2}} \rightarrow L^{s}}<\infty$, then $\left\|T_{\Omega}\right\|_{L^{p_{1} \times L^{p_{2}} \rightarrow L^{s}}}<\infty$. For some special domain $\Omega$, this property holds for triplets $\left(s, p_{1}, p_{2}\right)$ satisfying $\frac{1}{p_{1}}+\frac{1}{p_{2}}>\frac{1}{\min \{1, s\}}$.
\end{abstract}

2010 Mathematics Subject Classification: 42B25.

Key words: Integral restriction, multilinear operator.

\section{Introduction}

Let $T$ be a bilinear operator defined by

$$
T\left(f_{1}, f_{2}\right)(x)=\int_{\mathbb{R}^{2}} K\left(x, y_{1}, y_{2}\right) f_{1}\left(y_{1}\right) f_{2}\left(y_{2}\right) d y_{1} d y_{2}
$$

We consider its integral domain restriction operator (IDRO)

$$
T_{\Omega}\left(f_{1}, f_{2}\right)(x)=\int_{\Omega} K\left(x, y_{1}, y_{2}\right) f_{1}\left(y_{1}\right) f_{2}\left(y_{2}\right) d y_{1} d y_{2}
$$

where $\Omega$ is an open set in $\mathbb{R}^{2}$. We will show that $T_{\Omega}$ inherits the $L^{p_{1}} \times L^{p_{2}} \rightarrow L^{s}$ boundedness from the operator $T$, if $\left(s, p_{1}, p_{2}\right)$ satisfies $\frac{1}{p_{1}}+\frac{1}{p_{2}} \geq \frac{2}{\min \{1, s\}}$. For special domains $\Omega$, we can extend the range of $\left(s, p_{1}, p_{2}\right)$ to be $\frac{1}{p_{1}}+\frac{1}{p_{2}}>\frac{1}{\min \{1, s\}}$.

In order to study the stability of absolutely continuous spectrum for certain one-dimensional Schrödinger operator, in [1] Christ and Kiselev considered linear operators

$$
\left(K_{i} f\right)(\lambda)=\int_{\mathbb{R}^{+}} k_{i}(\lambda, x) f(x) d x, \quad i=1,2, \ldots, n,
$$


where the functions $k_{i}(\lambda, x), i=1,2 \ldots, n$ are defined on $I \times \mathbb{R}^{+}$and $I$ is a measurable set of $\mathbb{R}$, and the multilinear operator

$$
T_{n}\left(f_{1}, \ldots, f_{n}\right)(\lambda)=\int_{\mathbb{R}^{n}} \prod_{j=1}^{n} f_{j}\left(x_{j}\right) k_{j}\left(x_{j}, \lambda\right) \prod_{\alpha \in A} \chi_{\mathbb{R}^{+}}\left(x_{i_{\alpha}}-x_{i_{\alpha}^{\prime}}\right) d x
$$

where $A$ is any set of ordered pairs $\alpha=\left(i_{\alpha}, i_{\alpha}^{\prime}\right)$, with $1 \leq i_{\alpha}, i_{\alpha}^{\prime} \leq n$. Christ and Kiselev proved that if, for some $p \in[1,2)$ and $q>p$

$$
\left\|K_{i} f\right\|_{L^{q}(I, d \lambda)} \leq C_{i}\|f\|_{L^{p}\left(\mathbb{R}^{+}, d x\right)}, \quad i=1,2, \ldots, n,
$$

for all functions $f \in L^{p}\left(\mathbb{R}^{+}\right)$with compact supports, then

$$
\left\|T_{n}\left(f_{1}, f_{2}, \ldots, f_{n}\right)\right\|_{L^{s_{n}}} \leq C_{n} \prod_{i=1}^{n}\left\|f_{i}\right\|_{L^{p}},
$$

for all $f_{i} \in L^{p}\left(\mathbb{R}^{+}, d x\right)$ with $s_{n}^{-1}=n q^{-1}$. Especially, when $n=2$ and $\mathcal{A}=\{(1,2)\}$, we see

$$
T_{2}\left(f_{1}, f_{2}\right)(\lambda)=\int_{x_{1} \geq x_{2}} f_{1}\left(x_{1}\right) f_{2}\left(x_{2}\right) k_{1}\left(x_{1}, \lambda\right) k_{2}\left(x_{2}, \lambda\right) d x .
$$

Then Christ-Kiselev's result says that if for some $1 \leq p<2$ and $q>p$

$$
\left\|K_{i} f\right\|_{L^{q}} \leq C_{i}\|f\|_{L^{p}}, \quad i=1,2,
$$

then

$$
\left\|T_{2}\left(f_{1}, f_{2}\right)\right\|_{L^{q / 2}(\mathbb{R})} \leq C\left\|f_{1}\right\|_{L^{p}(\mathbb{R})}\left\|f_{2}\right\|_{L^{p}(\mathbb{R})},
$$

where $C$ depends on the constants $C_{1}$ and $C_{2}$.

A natural question raised is that, in the above restriction domain inequality, can we replace the domain $\left\{\left(x_{1}, x_{2}\right), x_{1} \geq x_{2}\right\}$ by any measurable set? More precisely, for a bilinear operator

$$
T\left(f_{1}, f_{2}\right)(x)=\int_{\mathbb{R}^{2}} K\left(x, y_{1}, y_{2}\right) f\left(y_{1}\right) f\left(y_{2}\right) d y_{1} d y_{2}
$$

we will study its IDRO

$$
T_{\Omega}\left(f_{1}, f_{2}\right)(x)=\int_{\Omega} K\left(x, y_{1}, y_{2}\right) f\left(y_{1}\right) f\left(y_{2}\right) d y_{1} d y_{2}
$$

for any measurable set $\Omega \subset \mathbb{R}^{2}$. Unlike the linear operator

$$
L(f)(x)=\int_{\mathbb{R}^{2}} K\left(x, y_{1}, y_{2}\right) f\left(y_{1}, y_{2}\right) d y_{1} d y_{2},
$$

$f\left(y_{1}, y_{2}\right)=f_{1}\left(y_{1}\right) f_{2}\left(y_{2}\right)$ is separable in the bilinear operator $T\left(f_{1}, f_{2}\right)$. This definition allows that $T_{\Omega}$ can inherit the $L^{p_{1}} \times L^{p_{2}} \rightarrow L^{s}$ boundedness from the operator $T$ for some triplets $\left(s, p_{1}, p_{2}\right)$.

We have the following theorem. 
Theorem 1.1. Let $T$ be the bilinear operator with kernel $K\left(x, y_{1}, y_{2}\right)$, that satisfies

$$
\left\|T\left(f_{1}, f_{2}\right)\right\|_{L^{s}(\mathbb{R})} \leq C_{1}\left\|f_{1}\right\|_{L^{p_{1}(\mathbb{R})}}\left\|f_{2}\right\|_{L^{p_{2}(\mathbb{R})}},
$$

for some constant $C_{1}$ and both $f_{i} \in L^{p_{i}}, i=1,2$, where $\left(s, p_{1}, p_{2}\right)$ satisfies $\frac{1}{p_{1}}+\frac{1}{p_{2}} \geq \frac{2}{\min \{1, s\}}$ and $0<s, p_{1}, p_{2}<\infty$. Then for any open subset $\Omega \subseteq \mathbb{R}^{2}$ and

$$
T_{\Omega}\left(f_{1}, f_{2}\right)(x)=\int_{\Omega} K\left(x, y_{1}, y_{2}\right) f_{1}\left(y_{1}\right) f_{2}\left(y_{2}\right) d y_{1} d y_{2},
$$

we have

$$
\left\|T_{\Omega}\left(f_{1}, f_{2}\right)\right\|_{L^{s}(\mathbb{R})} \leq C\left\|f_{1}\right\|_{L^{p_{1}(\mathbb{R})}}\left\|f_{2}\right\|_{L^{p_{2}(\mathbb{R})}},
$$

where $C$ only depends on $C_{1}$.

For a special domain, (1.15) is also true for $\left(s, p_{1}, p_{2}\right)$ which satisfies $\frac{1}{p_{1}}+\frac{1}{p_{2}}>\frac{1}{\min \{1, s\}}$ and $0<s, p_{1}, p_{2}<\infty$.

Let $\Psi$ be a family of sets $\Omega_{x}, x \in \mathbb{R}$ which satisfies the following conditions:

$$
\begin{array}{cl}
\emptyset \in \Psi \quad \text { and } & \mathbb{R} \in \Psi, \\
\text { for any } x<y, & \Omega_{x} \subseteq \Omega_{y} .
\end{array}
$$

We set $\Theta=\{\Psi: \Psi$ satisfies (1.16) and (1.17) $\}$, and set $\Sigma_{1}(\Psi)=\{(x, y) \in$ $\left.\mathbb{R}^{2}: y \in \Omega_{x}^{c}, \Omega_{x} \in \Psi\right\}, \Sigma_{2}(\Psi)=\left\{(x, y) \in \mathbb{R}^{2}: x \in \Omega_{y}^{c}, \Omega_{x} \in \Psi\right\}$. Let $\mathcal{A}$ be the algebra generated by some finite subset of $\cup_{\Psi \in \Theta}\left\{\Sigma_{1}(\Psi), \Sigma_{2}(\Psi)\right.$ : $\left.\Psi=\left\{\Omega_{x}\right\}_{x \in \mathbb{R}}\right\}$, then for any $\Sigma \in \mathcal{A}$ there exists $n$ such that $\Sigma$ can be written as finite unions and complements of sets in

$$
\left\{\Sigma_{1}\left(\Psi^{1}\right), \Sigma_{2}\left(\Psi^{1}\right), \ldots, \Sigma_{1}\left(\Psi^{n}\right), \Sigma_{2}\left(\Psi^{n}\right)\right\},
$$

with $\Psi^{k} \in \Theta$ for $k=1,2, \ldots, n$.

For the bilinear operator $T$, we consider its restriction operator

$$
T_{\Sigma}\left(f_{1}, f_{2}\right)(x)=\int_{\Sigma} K\left(x, y_{1}, y_{2}\right) f_{1}\left(y_{1}\right) f_{2}\left(y_{2}\right) d y_{1} d y_{2} \text {. }
$$

Theorem 1.2. Suppose $T$ is the bilinear operator defined in Theorem 1.1 and satisfies (1.13) for $\left(s, p_{1}, p_{2}\right)$ satisfying $\frac{1}{p_{1}}+\frac{1}{p_{2}}>\frac{1}{\min \{1, s\}}, 0<$ $s, p_{1}, p_{2}<\infty$. Then for $\Sigma \in \mathcal{A}$, there exists a constant $C>0$ dependent only on the domain and $C_{1}$ in (1.15), for any function $f_{i} \in L^{p_{i}}(\mathbb{R})$, $i=1,2$,

$$
\left.\| T_{\Sigma}\left(f_{1}, f_{2}\right)\right)\left\|_{L^{s}} \leq C\right\| f_{1}\left\|_{L^{p_{1}}}\right\| f_{2} \|_{L^{p_{2}}}
$$


We give some notations used in this paper. Let $\mathscr{S}:=\mathscr{S}\left(\mathbb{R}^{n}\right)$ be the Schwartz space. If $\Omega \subset \mathbb{R}^{n}$, then $|\Omega|$ denotes the Lebesgue measure of $\Omega$ and $\# \Omega$ denotes the cardinality of $\Omega$. For $\Omega_{1}, \Omega_{2} \subset \mathbb{R}^{n}$, notation $\Omega_{1} \subset$ a.e. $\Omega_{2}$ means that there exists a 0 measure set $A$ such that $\Omega_{1} \backslash A \subset \Omega_{2}$. We define $N(f)=\left\{x \in \mathbb{R}^{n}: f(x) \neq 0\right\}$ for any function $f$ defined on $\mathbb{R}^{n}$.

This paper is organized as follows. In Section 2, we give the proof for Theorem 1.1, by using dividing integral domains into rectangles. In Section 3, we prove Theorem 1.2. And in Section 4, we extend the results to high dimension and give corresponding results on special domains.

\section{Proof of Theorem 1.1}

In this section we give the proof of Theorem 1.1 by using inductive argument to divide the integral domains into domains on which the integral is controlled.

Since $T_{\Omega}$ is bilinear, we may assume without loss of generality throughout the proof that $\left\|f_{1}\right\|_{L^{p_{1}}}^{p_{1}}=\left\|f_{2}\right\|_{L^{p_{2}}}^{p_{2}}=1 / 2$ and $f_{i} \in \mathscr{S}(\mathbb{R}), i=1,2$. Let

$$
f(x)=\left|f_{1}(x)\right|^{p_{1}}+\left|f_{2}(x)\right|^{p_{2}} .
$$

Since

$$
T_{\Omega}\left(f_{1}, f_{2}\right)(x)=\int_{\Omega \cap(N(f) \times N(f))} K\left(x, y_{1}, y_{2}\right) f_{1}\left(y_{1}\right) f_{2}\left(y_{2}\right) d y_{1} d y_{2}
$$

If $|\Omega \cap(N(f) \times N(f))|=0$, then $T_{\Omega}\left(f_{1}, f_{2}\right)=0$. So we should consider the the nontrivial case $|\Omega \cap(N(f) \times N(f))| \neq 0$ and $\Omega \neq \mathbb{R}^{2}$. To estimate $T_{\Omega}\left(f_{1}, f_{2}\right)$, we need to decompose the support of the function $f$ into dyadic pieces and show that there exists a constant $C$, such that $\left\|T_{\Omega}\left(f_{1}, f_{2}\right)\right\|_{L^{s}} \leq C$.

We consider a partition of $\Omega \cap(N(f) \times N(f))$ into the dyadic pieces by the following inductive method. In this part, we denote $E(m, i, j)=$ $E(m, i) \times E(m, j)$ for simplicity.

Dividing process. Denote $E(0,1)=\mathbb{R}$.

Step 1: Find the smallest $x_{1,1} \in \overline{N(f)}$, such that

$$
\left\|f \chi_{E(1,1)}\right\|_{L^{1}}=\left\|f \chi_{E(1,2)}\right\|_{L^{1}}=\frac{1}{2} \text {. }
$$

Here $E(1,1)$ and $E(1,2)$ are defined as follows: If $x_{1,1}$ is an inner point of $N(f)$, then we define $E(1,1)=\left(-\infty, x_{1,1}\right)$ and $E(1,2)=\left[x_{1,1}, \infty\right)$; if $x_{1,1}$ is at the boundary, we define $E(1,1)=\left(-\infty, x_{1,1}\right)$ and $E(1,2)=$ $\left(x_{1,1}, \infty\right)$. We remark here since we let $f_{i} \in \mathscr{S}(\mathbb{R}), i=1,2, x_{1,1}$ exists and is unique. So we divide $E(0,1)$ into 2 intervals $E(1,1), E(1,2)$. 
Step $m$ : For each $E(m-1, i)$, we divide it into 2 intervals $E(m, 2 i-1)$, $E(m, 2 i)$ such that

$$
\left\|f \chi_{E(m, 2 i-1)}\right\|_{L^{1}}=\left\|f \chi_{E(m, 2 i)}\right\|_{L^{1}}=\frac{1}{2^{m}}
$$

for $1 \leq i \leq 2^{m-1}$ respectively.

\section{Selecting process.}

Step 1: Denote

$$
T_{1}=\{E(1, i, j): i, j=1,2\} .
$$

We drop the domains in $T_{1}$ which intersect $\Omega \cap(N(f) \times N(f))$ with 0 measure and let $D_{1}$ be the set of dropped domains in Step 1. So the dropped family in Step 1

$$
\begin{array}{r}
D_{1}=\left\{E(1, i, j) \in T_{1}:|E(1, i, j) \cap \Omega \cap(N(f) \times N(f))|=0,\right. \\
i, j=1,2\} .
\end{array}
$$

Then in $T_{1} \backslash D_{1}$ we select the domains which are contained a.e. in $\Omega \cap$ $(N(f) \times N(f))$, and let $S_{1}$ be the set of selected domains in Step 1. So the selected family in Step 1

$S_{1}=\left\{E(1, i, j) \in T_{1} \backslash D_{1}: E(1, i, j) \subset_{\text {a.e. }} \Omega \cap(N(f) \times N(f)), i, j=1,2\right\}$.

Denote the remaining domains in Step 1 by $R_{1}$, and we have $R_{1}=$ $T_{1} \backslash\left(S_{1} \cup D_{1}\right)$.

Step $m$ : Denote

$$
\begin{aligned}
T_{m}=\{ & E(m, 2 i-1,2 j-1), E(m, 2 i, 2 j-1), E(m, 2 i-1,2 j), E(m, 2 i, 2 j): \\
& \text { for any } \left.i, j=1,2, \ldots, 2^{m-1}, \text { such that } E(m-1, i, j) \in R_{m-1}\right\} .
\end{aligned}
$$

Also we drop the domains in $T_{m}$ which intersect $\Omega \cap(N(f) \times N(f))$ with 0 measure and let $D_{m}$ be the set of dropped domains in Step $m$. So the dropped family in Step $m$ is

$$
\begin{gathered}
D_{m}=\left\{E(m, i, j) \in T_{m}:|E(m, i, j) \cap(\Omega \cap(N(f) \times N(f)))|=0,\right. \\
\left.i, j=1,2,3, \ldots, 2^{m}\right\} .
\end{gathered}
$$

Then in $T_{m} \backslash D_{m}$ we select the domains which are almost everywhere contained in $\Omega \cap(N(f) \times N(f))$, and let $S_{m}$ be the set of selected domains in Step $m$. So the selected family in Step $m$ is

$$
\begin{aligned}
& S_{m}=\left\{E(m, i, j) \in T_{m} \backslash D_{m}: E(m, i, j) \subset_{\text {a.e. }} \Omega \cap(N(f) \times N(f)),\right. \\
& \left.i, j=1,2,3, \ldots, 2^{m}\right\} \text {. }
\end{aligned}
$$


Denote the remaining domains in Step $m$ by $R_{m}$, we have $R_{m}=T_{m}$ । $\left(S_{m} \cup D_{m}\right)$.

Next, we show that there is a 0 measure set $A \subset \cup_{m=1}^{\infty} \cup_{E(m, i, j) \in S_{m}}$ $E(m, i, j)$, such that

$$
\Omega \cap(N(f) \times N(f))=\bigcup_{m=1}^{\infty} \bigcup_{E(m, i, j) \in S_{m}} E(m, i, j) \backslash A
$$

and

$$
A \cap \Omega \cap(N(f) \times N(f))=\emptyset .
$$

According to our selecting process, there is a 0 measure set $A(m, i, j) \subset$ $E(m, i, j)$ and $A(m, i, j) \cap \Omega \cap(N(f) \times N(f))=\emptyset$ such that $E(m, i, j) \backslash$ $A(m, i, j) \subset \Omega \cap(N(f) \times N(f))$. We take $A=\cup_{m=1}^{\infty} \cup_{E(m, i, j) \in S_{m}} A(m, i, j)$, then we have

$$
\Omega \cap(N(f) \times N(f)) \supset \bigcup_{m=1}^{\infty} \bigcup_{E(m, i, j) \in S_{m}} E(m, i, j) \backslash A
$$

and

$$
A \cap \Omega \cap(N(f) \times N(f))=\emptyset .
$$

Now we show $\Omega \cap(N(f) \times N(f)) \subset \cup_{m=1}^{\infty} \cup_{E(m, i, j) \in S_{m}} E(m, i, j) \backslash A$. Since $N(f)$ is open, we have $\Omega \cap(N(f) \times N(f))$ is an open set. For all $x=\left(x_{1}, x_{2}\right) \in \Omega \cap(N(f) \times N(f))$, there exists a $\delta>0$, such that

$$
\left(x_{1}-\delta, x_{1}+\delta\right) \times\left(x_{2}-\delta, x_{2}+\delta\right) \subset \Omega \cap(N(f) \times N(f)) .
$$

And there exists $\varepsilon>0,\left\|f \chi_{\left(x_{1}-\delta, x_{1}+\delta\right)}\right\|_{L^{1}}>\varepsilon,\left\|f \chi_{\left(x_{2}-\delta, x_{2}+\delta\right)}\right\|_{L^{1}}>\varepsilon$. By the process of dividing, for the above $\delta>0$, there exist $N_{1}, N_{2}$, for all $n>N_{1}$ there exists $E\left(n, i_{n}\right)$ such that

$$
x_{1} \in E\left(n, i_{n}\right) \subset\left(x_{1}-\delta, x_{1}+\delta\right),
$$

and for all $n>N_{2}$ there exists $E\left(n, j_{n}\right)$ such that

$$
x_{2} \in E\left(n, j_{n}\right) \subset\left(x_{2}-\delta, x_{2}+\delta\right) .
$$

So for all $n>\max \left\{N_{1}, N_{2}\right\}$,

$$
\begin{aligned}
x=\left(x_{1}, x_{2}\right) \in E\left(n, i_{n}, j_{n}\right) \subset\left(x_{1}-\delta, x_{1}+\delta\right) & \times\left(x_{2}-\delta, x_{2}+\delta\right) \\
& \subset \Omega \cap(N(f) \times N(f)) .
\end{aligned}
$$

Remark 2.1. For $E(n, i, j)$ where $i, j \in\left\{1,2,3, \ldots, 2^{n}\right\}$, there exists a unique $E\left(k, i_{k}, j_{k}\right)$ such that $E(n, i, j) \subset E\left(k, i_{k}, j_{k}\right)$ for all $1 \leq k \leq$ $n, k \in \mathbb{Z}$. 
Proof: By the processes above, the existence is obvious. If there exist $E\left(k, i_{k}, j_{k}\right), E\left(k, \widetilde{i}_{k}, \widetilde{j}_{k}\right)$ such that $E(n, i, j) \subset E\left(k, i_{k}, j_{k}\right), E\left(k, \widetilde{i}_{k}, \widetilde{j}_{k}\right)$. Then $E(n, i, j) \subset E\left(k, i_{k}, j_{k}\right) \cap E\left(k, \widetilde{i}_{k}, \widetilde{j}_{k}\right) \neq \emptyset$, contradictory.

Then we choose any $n>\max \left\{N_{1}, N_{2}\right\}$ and claim the fact that there exist $E\left(k, i_{k}, j_{k}\right) \supset E\left(n, i_{n}, j_{n}\right)$, for some $k=1,2, \ldots, n$, such that $E\left(k, i_{k}, j_{k}\right) \in S_{k}$. If not, for $\left|E\left(1, i_{1}, j_{1}\right) \cap \Omega \cap(N(f) \times N(f))\right| \neq 0$, so $E\left(1, i_{1}, j_{1}\right) \notin D_{1}$ and $E\left(1, i_{1}, j_{1}\right)$ must be in $R_{1}$. Then we get $E\left(2, i_{2}, j_{2}\right) \in$ $T_{2}$, by the same argument, we get $E\left(2, i_{2}, j_{2}\right) \in R_{2}$. Step by step, finally we have $E\left(n, i_{n}, j_{n}\right) \in R_{n}$, this is contradictory, since $E\left(n, i_{n}, j_{n}\right) \subset$ $\Omega \cap(N(f) \times N(f))$.

According to our dividing and selecting, we get

$$
\begin{gathered}
\# S_{m}+\# D_{m}+\# R_{m}=\# T_{m}=4 \# R_{m-1}, \\
\quad \# T_{1}=4, \\
\# S_{m} \geq 0, \quad \# D_{m} \geq 0, \quad \# R_{m} \geq 0 .
\end{gathered}
$$

Since $\# R_{m} \leq 4 \# R_{m-1}, \# R_{1} \leq 4$, we get $\# R_{m} \leq 4^{m}$, then we have

$$
\sum_{k=1}^{m} \frac{1}{4^{k}}\left(\# S_{k}+\# D_{k}\right)=1-\frac{1}{4^{m}} \# R_{m} \leq 2 .
$$

Then we have the estimate for $T_{\Omega}\left(f_{1}, f_{2}\right)$ :

$$
\left|T_{\Omega}\left(f_{1}, f_{2}\right)\right| \leq \sum_{m=1}^{\infty} \sum_{E(m, i, j) \in S_{m}}\left|T_{\Omega}\left(f_{1} \chi_{E(m, i)}, f_{2} \chi_{E(m, j)}\right)\right| .
$$

We now consider two cases: $s \leq 1$ and $s>1$. Suppose first that $0<$ $s \leq 1$ and $\frac{1}{p_{1}}+\frac{1}{p_{2}} \geq \frac{2}{s}$. We use the fact $\left(\sum_{i=1}^{\infty}\left|A_{i}\right|\right)^{s} \leq \sum_{i=1}^{\infty}\left|A_{i}\right|^{s}$, where $A_{i} \in \mathbb{R}$. By our assumption we have $\left\|f_{i} \chi_{E_{m, l}}\right\|_{L^{p_{i}}}^{p_{i}} \leq\left\|f \chi_{E_{m, l}}\right\|_{L^{1}}=2^{-m}$, $i=1,2$. Then by (1.13) and (2.7), we have

$$
\begin{aligned}
\left\|T_{\Omega}\left(f_{1}, f_{2}\right)\right\|_{L^{s}}^{s} & \leq C_{1} \sum_{m=1}^{\infty} \sum_{E(m, i, j) \in S_{m}}\left\|f_{1} \chi_{E(m, i)}\right\|_{L^{p_{1}}}^{s}\left\|f_{2} \chi_{E(m, j)}\right\|_{L^{p_{2}}}^{s} \\
& \leq C_{1} \sum_{m=1}^{\infty} \# S_{m} 2^{-\frac{m s}{p_{1}}-\frac{m s}{p_{2}}} \\
& \leq C_{1} \sum_{m=1}^{\infty} \frac{1}{4^{m}}\left(\# S_{m}+\# D_{m}\right) 4^{m} 2^{-\frac{m s}{p_{1}}-\frac{m s}{p_{2}}} \\
& \leq C_{2}\left(\sup _{m \geq 1} 2^{m\left(2-s\left(\frac{1}{p_{1}}+\frac{1}{p_{2}}\right)\right)}\right) \sum_{m=1}^{\infty} \frac{1}{4^{m}}\left(\# S_{m}+\# D_{m}\right) \leq C_{3} .
\end{aligned}
$$


If $s>1$, by our assumption we have $\frac{1}{p_{1}}+\frac{1}{p_{2}} \geq 2$. Using Minkowski inequality on $L^{s}$ norm, we have:

$$
\begin{aligned}
\left\|T_{\Omega}\left(f_{1}, f_{2}\right)\right\|_{L^{s}} & \leq C_{4} \sum_{m=1}^{\infty} \sum_{E(m, i, j) \in S_{m}}\left\|f_{1} \chi_{E(m, i)}\right\|_{L^{p_{1}}}\left\|f_{2} \chi_{E(m, j)}\right\|_{L^{p_{2}}} \\
& \leq C_{4} \sum_{m=1}^{\infty} \# S_{m} 2^{-\frac{m}{p_{1}}-\frac{m}{p_{2}}} \\
& \leq C_{4} \sum_{m=1}^{\infty} \frac{1}{4^{m}}\left(\# S_{m}+\# D_{m}\right) 4^{m} 2^{-\frac{m}{p_{1}}-\frac{m}{p_{2}}} \\
& \leq C_{4} \sup _{m \geq 1}\left(2^{m\left(2-\left(\frac{1}{p_{1}}+\frac{1}{p_{2}}\right)\right)}\right) \sum_{m=1}^{\infty} \frac{1}{4^{m}}\left(\# S_{m}+\# D_{m}\right) \leq C_{5} .
\end{aligned}
$$

So, for both cases, there exists a constant $C$, such that $\left\|T_{\Omega}\left(f_{1}, f_{2}\right)\right\|_{L^{s}} \leq$ $C$. This completes the proof.

\section{Proof of Theorem 1.2}

In this section we give the proof of Theorem 1.2. To prove Theorem 1.2, we only need to show it is true for $\Sigma=\Sigma_{1}(\Psi)$ with $\Psi=$ $\left\{\Omega_{x}\right\}_{x \in \mathbb{R}} \in \Theta$.

First, we prove the theorem under an additional assumption that $\left|\Omega_{x}\right|$ (the Lebesgue measure of $\Omega_{x}$ ) depends continuously on $x$. We set $a=\sup _{\Omega_{x} \in \Psi}\left\{x: \Omega_{x}=\emptyset\right\}, b=\inf _{\Omega_{x} \in \Psi}\left\{x: \Omega_{x}=\mathbb{R}\right\}$. Here $a$ may be $-\infty$ and $b$ may be $+\infty$. Then

$$
\begin{aligned}
T_{\Sigma_{1}}\left(f_{1}, f_{2}\right)(x)= & \int_{\mathbb{R}} \int_{x_{2} \in \Omega_{x_{1}}^{c}} K\left(x, x_{1}, x_{2}\right) f_{1}\left(x_{1}\right) f_{2}\left(x_{2}\right) d x_{2} d x_{1} \\
= & \int_{a}^{b} \int_{x_{2} \in \Omega_{x_{1}}^{c}} K\left(x, x_{1}, x_{2}\right) f_{1}\left(x_{1}\right) f_{2}\left(x_{2}\right) d x_{2} d x_{1} \\
& +\int_{-\infty}^{a} \int_{\mathbb{R}} K\left(x, x_{1}, x_{2}\right) f_{1}\left(x_{1}\right) f_{2}\left(x_{2}\right) d x_{2} d x_{1} \\
= & I_{1}+I_{2} .
\end{aligned}
$$

For the boundedness of $I_{2}$, by the boundedness of $T$, we have

$$
\left\|I_{2}\right\| \leq\left\|T\left(f_{1} \chi_{(-\infty, a)}, f_{2}\right)\right\|_{L^{s}} \leq C_{1}\left\|f_{1}\right\|_{L^{p_{1}}}\left\|f_{2}\right\|_{L^{p_{2}}} .
$$

Next we consider the boundedness of $I_{1}$. Since $T_{\Sigma_{1}}$ is bilinear, we may assume without loss of generality throughout the proof that $\left\|f_{1}\right\|_{L^{p_{1}}}^{p_{1}}=$ 
$\left\|f_{2}\right\|_{L^{p_{2}}}^{p_{2}}=1 / 2$. Let

$$
f(x)=\left|f_{1}(x)\right|^{p_{1}}+\left|f_{2}(x)\right|^{p_{2}} .
$$

To estimate $I_{1}$ we need to decompose the support of the function $f$ into dyadic pieces and show that there exists a constant $C$, such that $\left\|I_{1}\right\|_{L^{s}} \leq C$. We consider a partition of $\mathbb{R}$ into the dyadic pieces in the following way:

$$
E_{m, j}=\Omega_{x_{m, j+1}} \backslash \Omega_{x_{m, j}},
$$

where

$$
x_{m, j}=\inf \left\{t: \int_{\Omega_{t}}|f(x)| d x=2^{-m} j\right\} .
$$

The value $x_{m, j}$ is well-defined for $j=1,2,3, \ldots, 2^{m}$ and $m \in Z^{+}$because $\left|\Omega_{x}\right|$ depends continuously on $x$. We denote by $M \subset \mathbb{R}^{2}$ the set

$$
M=\left\{\left(x_{1}, x_{2}\right) \in \mathbb{R}^{2}: x_{2} \in \Omega_{x_{1}}^{c}\right\} .
$$

Then we can claim that

$$
M \cap\left(\operatorname{supp}\left(f_{1}\right) \times \operatorname{supp}\left(f_{2}\right)\right)=\bigcup_{m=1}^{\infty} \bigcup_{\substack{l=1 \\ l \text { odd }}}^{2^{m}}\left(E_{m, l} \times E_{m, l+1}\right)
$$

(the proof is similar to the proof of Lemma 4.2 in [1] ). We begin to estimate $I_{1}$ :

$$
\left|I_{1}\right| \leq \sum_{m=1}^{\infty} \sum_{\substack{l=1 \\ l \text { odd }}}^{2^{m}}\left|T_{\Sigma_{1}}\left(f_{1} \chi_{E_{m, l}}, f_{2} \chi_{E_{m, l+1}}\right)\right| .
$$

We consider two different cases: $s \leq 1$ and $s>1$. Suppose first that $0<s \leq 1$. We use the fact $\left(\sum_{i=1}^{\infty}\left|A_{i}\right|\right)^{s} \leq \sum_{i=1}^{\infty}\left|A_{i}\right|^{s}$. By our assumption we have $\left\|f_{i} \chi_{E_{m, l}}\right\|_{L^{p_{i}}}^{p_{i}} \leq\left\|f \chi_{E_{m, l}}\right\|_{L^{1}}=2^{-m}, i=1,2$. Then, by (1.13),

$$
\begin{aligned}
\left\|I_{1}\right\|_{L^{s}}^{s} & \leq C_{1} \sum_{m=1}^{\infty} \sum_{\substack{l=1 \\
l \text { odd }}}^{2^{m}}\left\|f_{1} \chi_{E_{m, l}}\right\|_{L^{p_{1}}}^{s}\left\|f_{2} \chi_{E_{m, l+1}}\right\|_{L^{p_{2}}}^{s} \\
& \leq C_{1} \sum_{m=1}^{\infty} \sum_{\substack{l=1 \\
l \text { odd }}}^{2^{m}} 2^{-\frac{m s}{p_{1}}-\frac{m s}{p_{2}}} \\
& \leq C_{2} \sum_{m=1}^{\infty} 2^{m\left(1-s\left(\frac{1}{p_{1}}+\frac{1}{p_{2}}\right)\right)} \leq C_{3}
\end{aligned}
$$


If $s>1$, by our assumption we have $\frac{1}{p_{1}}+\frac{1}{p_{2}}>1$. Using Minkowski inequality on $L^{s}$ norm, we have:

$$
\begin{aligned}
\left\|I_{1}\right\|_{L^{s}} & \leq C_{4} \sum_{m=1}^{\infty} \sum_{\substack{l=1 \\
l \text { odd }}}^{2^{m}}\left\|f_{1} \chi_{E_{m, l}}\right\|_{L^{p_{1}}}\left\|f_{2} \chi_{E_{m, l+1}}\right\|_{L^{p_{2}}} \\
& \leq C_{4} \sum_{m=1}^{\infty} \sum_{\substack{l=1 \\
l \text { odd }}}^{2^{m}} 2^{-\frac{m}{p_{1}}} 2^{-\frac{m}{p_{2}}} \\
& \leq C_{5} \sum_{m=1}^{\infty} 2^{m\left(1-\frac{1}{p_{1}}-\frac{1}{p_{2}}\right)} \leq C_{6} .
\end{aligned}
$$

So, in either case, there exists a constant $C$, such that $\left\|I_{1}\right\|_{L^{s}} \leq C$. This completes the proof under the assumption that $\left|\Omega_{x}\right|$ is a continuous function of $x$.

Then we consider the general case. We use the same method that Kiselev used in $[\mathbf{3}]$ when dealing with the a.e. convergence of integral operators.

We consider the function $\left|\Omega_{x}\right|$. We say there is a jump for $\Omega_{x}$ at $x$ if $\lim _{x \rightarrow x_{0_{+}}}\left|\Omega_{x}\right| \neq\left|\Omega_{x_{0}}\right|$ or $\lim _{x \rightarrow x_{0_{-}}}\left|\Omega_{x}\right| \neq\left|\Omega_{x_{0}}\right|$. We denote the value of jump by $h_{ \pm}$:

$$
h_{+}(x)=\left|\Omega_{x+0} \backslash \Omega_{x}\right|, \quad h_{-}(x)=\left|\Omega_{x} \backslash \Omega_{x-0}\right| .
$$

Since $\left|\Omega_{x}\right|$ is monotone, according to our assumption $\Omega_{x} \subset \Omega_{y}$ for $x<y$. The set of values of $h_{ \pm}$where any jump may occur is at most countable. Let $\left\{x_{n}\right\}_{n=1}^{\infty}$ be the sequence of these points. If for some $x$, both $h_{+}$ and $h_{-}$are nonzero, let $x_{n}=x_{n+1}=x$, for some $n$. We set $\Delta_{x_{n}}=$ $\Omega_{x_{n}+0} \backslash \Omega_{x_{n}}$, if $\left|\Omega_{x_{n}+0} \backslash \Omega_{x}\right| \neq 0$, and $\Delta_{x_{n}}=\Omega_{x_{n}} \backslash \Omega_{x_{n}-0}$ otherwise, and set the new measure space:

$$
\Theta=\Theta_{0} \cup\left(\bigcup_{m} \Theta_{m}\right)
$$

in which $\Theta_{0}=\left(\mathbb{R} \backslash \cup_{n} \Delta_{x_{n}}\right) \times\{0\}$ and $\Theta_{m}=\Delta_{x_{m}} \times[0,1]$. While on $\Theta_{m}$ the measure $\mu$ equals the product measure $d x \times d \nu$ ( $d \nu$ being a Lebesgue measure on $[0,1])$ and on $\Theta_{0}, d \mu=d x \times d \delta(0)$.

Then we construct the new bilinear operator:

$$
\widetilde{T}\left(\widetilde{f}_{1}, \widetilde{f}_{2}\right)(x)=\int_{\Theta^{2}} \widetilde{K}\left(x, \mathrm{y}_{1}, \mathrm{y}_{2}\right) \widetilde{f}_{1}\left(\mathrm{y}_{1}\right) \widetilde{f}_{2}\left(\mathrm{y}_{2}\right) d \mu\left(\mathrm{y}_{1}, \mathrm{y}_{2}\right),
$$

where $\widetilde{K}\left(x, \mathrm{y}_{1}, \mathrm{y}_{2}\right)$ is a new kernel defined on $\mathbb{R} \times \Theta \times \Theta$, which is equal to $K\left(x, x_{1}, x_{2}\right)$ for all $x$, when $\left(\mathrm{y}_{1}, \mathrm{y}_{2}\right)=\left(x_{1}, 0, x_{2}, 0\right) \in \Theta_{0} \times \Theta_{0}$. If 
$\left(\mathrm{y}_{1}, \mathrm{y}_{2}\right)=\left(x_{1}, y_{1}, x_{2}, y_{2}\right) \in \Theta_{x_{n}} \times \Theta_{x_{m}}$, which is $\left(y_{1}, y_{2}\right) \in[0,1] \times$ $[0,1]$ and $\left(x_{1}, x_{2}\right) \in \Delta_{n} \times \Delta_{m}$ for some $n$ and $m$, then $\widetilde{K}\left(x, \mathrm{y}_{1}, \mathrm{y}_{2}\right)=$ $K\left(x, x_{1}, x_{2}\right)$ for all $x$. If $\left(\mathrm{y}_{1}, \mathrm{y}_{2}\right)=\left(x_{1}, 0, x_{2}, y_{2}\right) \in \Theta_{0} \times \Theta_{n}$, which is $\left(x_{1}, x_{2}\right) \in\left(\mathbb{R} \backslash \cup_{n} \Delta_{x_{n}}\right) \times \Delta_{x_{n}}$ for some $n$, then $\widetilde{K}\left(x, \mathrm{y}_{1}, \mathrm{y}_{2}\right)=K\left(x, x_{1}, x_{2}\right)$ for all $x$. If $\left(\mathrm{y}_{1}, \mathrm{y}_{2}\right)=\left(x_{1}, y_{1}, x_{2}, 0\right) \in \Theta_{n} \times \Theta_{0}$, which is $\left(x_{1}, x_{2}\right) \in$ $\Delta_{x_{n}} \times\left(\mathbb{R} \backslash \cup_{n} \Delta_{x_{n}}\right)$ for some $n$, then $\widetilde{K}\left(x, \mathrm{y}_{1}, \mathrm{y}_{2}\right)=K\left(x, x_{1}, x_{2}\right)$ for all $x$.

Next, define a family $\widetilde{\Omega}_{u}$ of the extending measurable sets in $\Theta$. We construct $\widetilde{\Omega}_{u}$ so that $\mu\left(\widetilde{\Omega}_{u}\right)=u$ which means $\mu\left(\widetilde{\Omega}_{u}\right)$ is continuous on $u$.

Let $x_{0}(u)=\sup _{t}\left\{t:\left|\Omega_{t}\right| \leq u\right\}$. If $x_{0}(u) \neq x_{n}$ for any $n$, we let

$$
\widetilde{\Omega}_{u}=\left(\left(\Omega_{x_{0}(u)} \times\{0\}\right) \cap \Theta_{0}\right) \cup\left(\bigcup_{x_{m}<x_{0}(u)} \Theta_{m}\right) .
$$

If at $x_{0}(u)$ we have a jump on the left, we let

$$
\begin{aligned}
\widetilde{\Omega}_{u}=\left(\left(\Omega_{x_{0}(u)-0} \times\{0\}\right) \cap \Theta_{0}\right) \cup & \left(\bigcup_{x_{m}<x_{0}(u)} \Theta_{m}\right) \\
& \cup\left(\Delta_{x_{n}} \times\left[0, \frac{u-\left|\Omega_{x_{0}(u)-0}\right|}{\left|\Omega_{x_{0}(u)}\right|}\right]\right) .
\end{aligned}
$$

If at $x_{0}(u)$ we have a jump on the right, we let

$\widetilde{\Omega}_{u}=\left(\left(\Omega_{x_{0}(u)} \times\{0\}\right) \cap \Theta_{0}\right) \cup\left(\bigcup_{x_{m}<x_{0}(u)} \Theta_{m}\right) \cup\left(\Delta_{x_{n}} \times\left[0, \frac{u-\left|\Omega_{x_{0}(u)}\right|}{\left|\Omega_{x_{0}(u)}\right|}\right]\right)$.

We can give the following claim without proof (details can be found in $[\mathbf{2}])$ :

(1) Let $\widetilde{\Psi}$ be a family of sets $\widetilde{\Omega}_{u}$, then $\emptyset \in \widetilde{\Psi}, \Theta \in \widetilde{\Psi}$, and $\widetilde{\Omega}_{u_{1}} \subseteq \widetilde{\Omega}_{u_{2}}$ for any $u_{1}<u_{2}$.

(2) If we let $u(x)=\sup _{t \leq x}\left\{\left|\Omega_{t}\right|\right\}$, and for given $f_{i} \in L^{p_{i}}(\mathbb{R})$, let $\widetilde{f}_{i}(\widetilde{x})=$ $f_{i}(x)$ when $(x, 0) \in \Theta_{0}$ and $\tilde{f}_{i}(\widetilde{x})=f_{i}(x)$ in which $\widetilde{x}=(x, y)$ for any $y \in[0,1]$ if $x \in \Delta_{x_{n}}$ for some $n$, then we have

$$
\iint_{u\left(y_{2}\right) \in \widetilde{\Omega}_{u\left(y_{1}\right)}^{c}} \widetilde{K}\left(x, \widetilde{y}_{1}, \widetilde{y}_{2}\right) \widetilde{f}_{1}\left(\widetilde{y}_{1}\right) \widetilde{f}_{2}\left(\widetilde{y}_{2}\right) d \mu\left(\widetilde{y}_{1}, \widetilde{y}_{2}\right)=T_{\Sigma_{1}}\left(f_{1}, f_{2}\right) .
$$

(3) There exists a constant $C$, such that

$$
\left\|\widetilde{T}\left(\widetilde{f}_{1}, \widetilde{f}_{2}\right)\right\|_{L^{s}(\mathbb{R})} \leq C_{1}\left\|\widetilde{f}_{1}\right\|_{L^{p_{1}(\Theta, d \mu)}}\left\|\widetilde{f}_{2}\right\|_{L^{p_{2}(\Theta, d \mu)}} .
$$

Hence, we have shown the bound (1.19) in general case. 


\section{Some corollaries and remarks}

In this section, we will show some corollaries and remarks.

Remark 4.1. For a multilinear operator

$$
\begin{aligned}
& T\left(f_{1}, f_{2}, \ldots, f_{n}\right)(x) \\
& =\int_{\mathbb{R}^{n}} K\left(x, y_{1}, y_{2}, \ldots, y_{n}\right) f_{1}\left(y_{1}\right) f_{2}\left(y_{2}\right) \cdots f_{n}\left(y_{n}\right) d y_{1} d y_{2} \cdots d y_{n}
\end{aligned}
$$

we assume that it satisfies

$$
\left\|T\left(f_{1}, f_{2}, \ldots, f_{n}\right)\right\|_{L^{s}} \leq C \prod_{k=1}^{n}\left\|f_{k}\right\|_{L^{p_{k}}},
$$

where $\left(s, p_{k}\right), k=1,2, \ldots, n$, satisfy:

$$
0<s, p_{k}<\infty, \sum_{k=1}^{n} \frac{1}{p_{k}} \geq n \max \left\{\frac{1}{s}, 1\right\} .
$$

Let $\Omega \in \mathbb{R}^{n}$ be any open set, and

$$
\begin{aligned}
& T_{\Omega}\left(f_{1}, f_{2}, \ldots, f_{n}\right)(x) \\
& =\int_{\Omega} K\left(x, y_{1}, y_{2}, \ldots, y_{n}\right) f_{1}\left(y_{1}\right) f_{2}\left(y_{2}\right) \cdots f_{n}\left(y_{n}\right) d y_{1} d y_{2} \cdots d y_{n},
\end{aligned}
$$

we have

$$
\left\|T_{\Omega}\left(f_{1}, f_{2}, \ldots, f_{n}\right)\right\|_{L^{s}} \leq C_{1} \prod_{k=1}^{n}\left\|f_{k}\right\|_{L^{p_{k}}},
$$

where $C_{1}$ depends only on the $\|T\|_{L^{p_{1}} \times L^{p_{2}} \times \cdots \times L^{p_{k}} \rightarrow L^{s}}$ norm.

We can see that the special case in $[\mathbf{1}](\{(x, y), x \geq y\})$ satisfies the condition required for the region in Theorem 1.2 even though it looks complicated. Also, there are some other special cases, such as convex sets.

Remark 4.2. For $k=1,2$, let $f_{k} \in L^{p_{k}}\left(I_{k}\right)$ and $I_{k}$ be measurable sets in $\mathbb{R}$. Assume that $\left(s, p_{1}, p_{2}\right)$ satisfies $\frac{1}{p_{1}}+\frac{1}{p_{2}}>\frac{1}{\min \{1, s\}}$ and $0<s, p_{1}, p_{2}<\infty$. If the whole space $\mathbb{R} \in \Psi$ in the first condition (1.16) is replaced by $I_{1}, I_{2} \in \Psi$, then (1.15) and (1.19) also holds. 
Corollary 4.3. Let $T$ be as in Theorem 1.2, $\left(s, p_{1}, p_{2}\right)$ satisfies $\frac{1}{p_{1}}+\frac{1}{p_{2}}>$ $\frac{1}{\min \{1, s\}}$ and $0<s, p_{1}, p_{2}<\infty$. Then for any function $f_{i} \in L^{p_{i}}(\mathbb{R})$, $i=1,2$,

$$
\left\|T_{B}\left(f_{1}, f_{2}\right)\right\|_{L^{s}} \leq C \prod_{i=1}^{2}\left\|f_{i}\right\|_{L^{p_{i}}},
$$

where $T_{B}$ is given by

$$
T_{B}\left(f_{1}, f_{2}\right)(x)=\int_{B(0,1)} K\left(x, y_{1}, y_{2}\right) f_{1}\left(y_{1}\right) f_{2}\left(y_{2}\right) d y .
$$

The constant $C$ depends only on the constants in the norm bound (1.13) for the operator $T$.

Proof: We set $g_{k}=f_{k} \chi_{[-1,1]}, i=1,2$. We divide the ball $B(0,1)=$ $\left\{\left(y_{1}, y_{2}\right): y_{1}^{2}+y_{2}^{2}<1\right\}$ into two parts $B_{+}=\left\{\left(y_{1}, y_{2}\right): y_{1} \in[0,1]\right.$, $\left.y_{2} \in\left(-\sqrt{1-y_{1}^{2}}, \sqrt{1-y_{1}^{2}}\right)\right\}$ and $B_{-}=\left\{\left(y_{1}, y_{2}\right): y_{1} \in[-1,0], y_{2} \in\right.$ $\left.\left(-\sqrt{1-y_{1}^{2}}, \sqrt{1-y_{1}^{2}}\right)\right\}$.

Let $\Phi_{+}=\left\{\Omega_{x}\right\}_{x \in[0,1]}$ with $\Omega_{x}=\left(-1,-\sqrt{1-x^{2}}\right] \cup\left[\sqrt{1-x^{2}}, 1\right)$ for $x \in[0,1]$. Then $\emptyset=\Omega_{0} \in \Phi_{+},(-1,1)=\Omega_{1} \in \Phi_{+}$and for $x<y$, $\Omega_{x} \subset \Omega_{y}$.

Let $\Phi_{-}=\left\{\widetilde{\Omega}_{x}\right\}_{x \in[-1,0]}$ with $\widetilde{\Omega}_{x}=\left(-\sqrt{1-x^{2}}, \sqrt{1-x^{2}}\right)$. Then $\emptyset=$ $\widetilde{\Omega}_{-1} \in \Phi_{-},(-1,1)=\widetilde{\Omega}_{0} \in \Phi_{-}$and for $x<y, \widetilde{\Omega}_{x} \subset \widetilde{\Omega}_{y}$. Since

$$
\begin{aligned}
T_{B}\left(f_{1}, f_{2}\right)(x)= & \int_{B(0,1)} K\left(x, y_{1}, y_{2}\right) f_{1}\left(y_{1}\right) f_{2}\left(y_{2}\right) d y \\
= & \int_{B_{+}} K\left(x, y_{1}, y_{2}\right) f_{1}\left(y_{1}\right) f_{2}\left(y_{2}\right) d y \\
& +\int_{B_{-}} K\left(x, y_{1}, y_{2}\right) f_{1}\left(y_{1}\right) f_{2}\left(y_{2}\right) d y \\
= & \int_{y_{2} \in \Omega_{y_{1}}^{c}, y_{1} \in[0,1]} K\left(x, y_{1}, y_{2}\right) g_{1}\left(y_{1}\right) g_{2}\left(y_{2}\right) d y \\
& +\int_{-1}^{1} \int_{-1}^{0} K\left(x, y_{1}, y_{2}\right) g_{1}\left(y_{1}\right) g_{2}\left(y_{2}\right) d y_{1} d y_{2} \\
& -\int_{y_{2} \in \widetilde{\Omega}_{y_{1}}^{c}, y_{1} \in[-1,0]} K\left(x, y_{1}, y_{2}\right) g_{1}\left(y_{1}\right) g_{2}\left(y_{2}\right) d y .
\end{aligned}
$$

So by using Theorem 1.2 and Remark 4.2, we get (4.6). 
The proof gives us a method of dealing with some area whose boundary consists of finite monotonic functions and lines parallel to coordinate axes.

Corollary 4.4. Let the bilinear operator $T$ with kernel $K\left(x, y_{1}, y_{2}\right)$ be as in Theorem 1.2 with $\left(s, p_{1}, p_{2}\right)$ satisfying $\frac{1}{p_{1}}+\frac{1}{p_{2}}>\frac{1}{\min \{1, s\}}$ and $0<s, p_{1}, p_{2}<\infty$. For a convex bounded measurable set $\mathcal{K} \subseteq \mathbb{R}^{2}, T_{\mathcal{K}}$ is given by

$$
T_{\mathcal{K}}\left(f_{1}, f_{2}\right)(x)=\int_{\mathcal{K}} K\left(x, y_{1}, y_{2}\right) f_{1}\left(y_{1}\right) f_{2}\left(y_{2}\right) d y .
$$

Then we have

$$
\left\|T_{\mathcal{K}}\left(f_{1}, f_{2}\right)\right\|_{L^{s}} \leq C \prod_{i=1}^{2}\left\|f_{i}\right\|_{L^{p_{i}}} .
$$

Finally we make some remarks of extending our theorem to high dimensional cases $n \geq 1$. The extension of Theorem 1.2 is involved. The main difficulty is how to define an order for sets $\Omega_{x}$ and $\Omega_{y}$ where $x, y \in \mathbb{R}^{n}$. To this end, we should give an order for points in higher dimension. A lot of orders can be given. In this paper, we just show two typical cases which can be proved in the same method.

We consider the bounded bilinear operator $T: L^{p_{1}} \times L^{p_{2}} \rightarrow L^{s}$ given by

$$
T\left(f_{1}, f_{2}\right)(\lambda)=\int K\left(\lambda, x_{1}, x_{2}\right) f_{1}\left(x_{1}\right) f_{2}\left(x_{2}\right) d x,
$$

and assume

$$
\left\|T\left(f_{1}, f_{2}\right)\right\|_{L^{s}} \leq C_{1}\left\|f_{1}\right\|_{L^{p_{1}}}\left\|f_{2}\right\|_{L^{p_{2}}} .
$$

Let $\Psi$ be a family of sets $\Omega_{x}$ and $\kappa_{1}(x)=|x|, \kappa_{2}(x)=\sum_{i=1}^{n} x_{i}$, which satisfies the following conditions:

(1) $\emptyset \in \Psi$ and $\mathbb{R}^{n} \in \Psi$;

(2) for any $\kappa_{1}(x)<\kappa_{1}(y)$ or $\kappa_{2}(x)<\kappa_{2}(y), \Omega_{x} \subseteq \Omega_{y}$.

Set $\Theta=\{\Psi, \Psi$ satisfies (1) and (2) $\}, \Sigma_{1}(\Psi)=\left\{(x, y) \in \mathbb{R}^{2}: y \in \Omega_{x}^{c}\right\}$, and $\Sigma_{2}(\Psi)=\left\{(x, y) \in \mathbb{R}^{2}: x \in \Omega_{y}^{c}\right\}$. Let $\Sigma$ be any set in $\mathcal{A}$, where $\mathcal{A}$ is the algebra generated by some finite subset of $\cup_{\Psi \in \Theta}\left\{\Sigma_{1}(\Psi), \Sigma_{2}(\Psi)\right\}$. Consider the bilinear operator $T_{2}$ given by

$$
T_{2}\left(f_{1}, f_{2}\right)(\lambda)=\int_{\Sigma} K\left(\lambda, x_{1}, x_{2}\right) f_{1}\left(x_{1}\right) f_{2}\left(x_{2}\right) d x_{1} d x_{2} .
$$


Remark 4.5. For any function $f_{i} \in L^{p_{i}}\left(\mathbb{R}^{n}\right), i=1,2$, and $\left(s, p_{1}, p_{2}\right)$ satisfying $\frac{1}{p_{1}}+\frac{1}{p_{2}}>\frac{1}{\min \{1, s\}}$ and $0<s, p_{1}, p_{2}<\infty$, if (4.11) holds, then

$$
\left\|T_{2}\left(f_{1}, f_{2}\right)\right\|_{L^{s}} \leq C \prod_{i=1}^{2}\left\|f_{i}\right\|_{L^{p_{i}}} .
$$

The constant $C$ depends only on the constant $C_{1}$ in (4.11) and the domain.

At last we remark that the relationship of $\left(p_{1}, p_{2}, s\right)$ in Theorem 1.2 is optimal in some sense.

Remark 4.6. Here we refer to Muscalu et al. [4]. Let

$$
T\left(f_{1}, f_{2}\right)(x)=\int_{\mathbb{R}^{2}} e^{-2 \pi i\left(x_{2}-x_{1}\right) x} f_{1}\left(x_{1}\right) f_{2}\left(x_{2}\right) d x_{1} d x_{2}=\widehat{f}_{1}(-x) \widehat{f}_{2}(x) .
$$

Here $\widehat{f}$ is the Fourier transform of $f$. Let $\Sigma=\left\{\left(x_{1}, x_{2}\right) \in \mathbb{R}^{2}, x_{1}<x_{2}\right\}$ and

$$
T_{\Sigma}\left(f_{1}, f_{2}\right)(x)=\int_{x_{1}<x_{2}} e^{-2 i\left(x_{2}-x_{1}\right) x} f_{1}\left(x_{1}\right) f_{2}\left(x_{2}\right) d x_{1} d x_{2} .
$$

Actually one may find that if we choose $f_{1}=f_{2}$, then $T_{\Sigma}\left(f_{1}, f_{1}\right)(x)$ is essentially $P\left(\left|\widehat{f}_{1}\right|^{2}\right)(x)$. Here $\widehat{P(f)}=\chi_{(-\infty, 0]} \widehat{f}=\frac{1}{2}(I-i H)\left(\left|\widehat{f}_{1}\right|^{2}\right)$ and $H$ is the Hilbert transform. Thus although we have

$$
\left\|T\left(f_{1}, f_{2}\right)\right\|_{L^{1}} \leq C\left\|\widehat{f}_{1}\right\|_{L^{2}}\left\|\widehat{f}_{2}\right\|_{L^{2}} \leq C\left\|f_{1}\right\|_{L^{2}}\left\|f_{2}\right\|_{L^{2}}
$$

it fails that

$$
\left\|T_{\Sigma}\left(f_{1}, f_{2}\right)\right\|_{L^{1}} \leq C\left\|f_{1}\right\|_{L^{2}}\left\|f_{2}\right\|_{L^{2}} .
$$

Since the $L^{1}$ boundedness of Hilbert transform fails.

Acknowledgments. Meng Wang is partially supported by NSFC 11371316. Weiren Zhao is partially supported by NSFC 11571306.

\section{References}

[1] M. Christ And A. Kiselev, Absolutely continuous spectrum for one-dimensional Schrödinger operators with slowly decaying potentials: some optimal results, J. Amer. Math. Soc. 11(4) (1998), 771-797. DOI : 10.1090/S0894-0347-98-00276-8.

[2] M. Christ And A. Kiselev, Maximal functions associated to filtrations, J. Funct. Anal. 179(2) (2001), 409-425. DOI: 10.1006/ jfan.2000.3687. 
[3] A. Kiselev, An interpolation theorem related to the a.e. convergence of integral operators, Proc. Amer. Math. Soc. 127(6) (1999), 1781-1788. DOI : 10.1090/S0002-9939-99-04681-X.

[4] C. Muscalu, T. TaO, and C. Thiele, A counterexample to a multilinear endpoint question of Christ and Kiselev, Math. Res. Lett. 10(2-3) (2003), 237-246. DOI: 10.4310/MRL.2003.v10.n2.a10.

Weiren Zhao and Meng Wang:

Department of Mathematics

Zhejiang University

310027, Hangzhou

P. R. China

E-mail address: zjzjzwr@126.com

E-mail address: mathdreamcn@zju.edu.cn

Guoping Zhao:

School of Applied Mathematics

Xiamen University of Technology

Xiamen, 361024

P. R. China

E-mail address: guopingzhaomath@gmail.com

Primera versió rebuda el 3 de novembre de 2014, darrera versió rebuda el 21 de gener de 2016. 